\section{Retinopatía Diabética en Chile: un reporte cuantitativo de la proporción de diabéticos con fondo de ojo anual}

\author{
ROLANDO SILVA-JORQUERA ${ }^{1, \mathrm{a}, \mathrm{b}}$, CLAUDIO ZETT $^{1, \mathrm{a}, \mathrm{c}}$
}

\section{Coverage rates of eye fundus examinations among Chilean patients with diabetes mellitus}

Background: Timely eye fundus examinations are essential to prevent the consequences of retinopathy among patients with type 2 diabetes mellitus. Aim: To assess the coverage rate (CR) of eye fundus examination in the Chilean diabetic population, between 2011 and 2019. Material and Methods: Analysis of monthly statistical summaries of the Cardiovascular Health Program published online by the Chilean Ministry of Health. The number of patients aged 15 years or more with a diagnosis of Type 2 diabetes mellitus and the number of those who had an eye fundus examination within the same year, were obtained. Data was analyzed at a national and regional level. Results: The national eye fundus examination CR was 19.1\% in 2011. In 2016, the figures became significantly higher, reaching 32.5\%. In 2019, the highest value of $36.5 \%$ was recoded although not significantly different from 2016. The highest average annual CR was observed in Nuble Health Service (49.5\%), and the lowest in Central Metropolitan Service (15\%). The highest CR positive absolute and relative variation between 2011 and 2019 was observed in Viña del Mar Quillota Health Service (38.9 and $489 \%$ respectively), and the lowest negative variation was observed in Araucania Norte Health Service (-8.42 and $-24.21 \%$ respectively). Conclusions: There is a low eye fundus examination $C R$ in Chile, with important differences between regional health services.

(Rev Med Chile 2021; 149: 971-979)

Key words: Blindness; Delivery of Health Care; Diabetes Mellitus; Diabetic Retinopathy; Diagnostic Screening Programs.
'Pontificia Universidad Católica de Valparaíso, Valparaíso, Chile.

aTecnólogo Médico.

bMáster en Investigación Clínica-Salud Internacional. ${ }^{\mathrm{CPhD}}$ en Oftalmología y Ciencias Visuales.

Trabajo no recibió

financiamiento.

Los autores declaran no tener

conflictos de interés.

Recibido el 26 de septiembre de 2020, aceptado el 6 de abril de 2021

Correspondencia a: Rolando Silva Jorquera Avenida Brasil 2950, Valparaíso, Chile.

rolando.silva@pucv.cl
J unto con el aumento de la esperanza de vida ${ }^{1}$, también lo hace la prevalencia de la Diabetes Mellitus (DM), estableciéndose como la tercera causa de muerte en el mundo ${ }^{2}$. Esta enfermedad afecta a 463 millones de personas ${ }^{3}$, estimándose que $30 \%$ desarrollará retinopatía diabética $(\mathrm{RD})^{4}$. El tratamiento oportuno mediante fotocoagulación ${ }^{5}$ en complemento de inyecciones intravítreas ${ }^{6}$ y cirugía vitreoretinal para los casos más avanza$\mathrm{dos}^{7}$, reducen el riesgo de pérdida visual y/o cegue$\mathrm{ra}^{8}$. Las estimaciones más actuales de prevalencia de RD en Chile van desde $14,9 \%{ }^{9}$ a $24,78 \%{ }^{10,11}$. La DM (tipo I y II) y la RD son enfermedades cubiertas por las Garantías Explícitas en Salud (GES) ${ }^{12}$, financiada a través del Fondo Nacional de Salud, la cual cubre a 14.244 .833 personas el año $2019^{13}$; un $74,6 \%$ de la población.

Existe una guía clínica de RD desarrollada por el Ministerio de Salud (MINSAL) que sugiere una evaluación anual de fondo de ojo (FO) realizado por un médico oftalmólogo o través de una evaluación retinográfica. Las retinografías son captu- 
radas a nivel primario por un Tecnólogo Médico especialista de Oftalmología (TMO) mediante una cámara fotográfica retinal no midriática ${ }^{14}$. Esta última modalidad teleoftalmológica ha optimizado el tamizaje, generando pertinencia en la derivación, reduciendo la sobrecarga sanitaria a nivel secundario y terciario ${ }^{15} \mathrm{y}$ aumentando la cobertura del tamizaje de $\mathrm{FO}^{11}$.

Entre los objetivos de la estrategia nacional de salud del MINSAL, se establece disminuir la mortalidad asociada a DM, mejorando la calidad de atención de las personas diabéticas. Para ello se fija como meta $80 \%$ en la tasa de cobertura (TC) de FO para los grupos etáreos de 15 a 24 años y mayores de 65 , mientras que, para la población entre 25 y 64 años, se considera una meta de $90 \%{ }^{16}$. Inglaterra logró recientemente una TC similar, alcanzando $82,7 \%{ }^{17}$.

Chile cuenta con 29 Servicios de Salud (SS), los cuales son los encargados de ejecutar los FO en las 346 comunas existentes en todo el país, siendo realizado principalmente en las Unidades de Atención Primaria Oftalmológica (UAPO).

No existen publicaciones oficiales acerca de las TC de FO nacional, con excepción de 32,4\% alcanzada en el 2016 según el sitio web del MINSAL. ${ }^{18}$ Tampoco se han establecido las TC desagregadas por SS ni por comunas.

El propósito de esta investigación es determinar y analizar la TC anual de FO en Chile en cada uno de sus SS entre los años 2011 y 2019, permitiendo evaluar la realidad nacional con evidencia estadística, como soporte para el desarrollo y mejoramiento de políticas públicas de salud en Chile.

\section{Materiales y Métodos}

Estudio analítico, cuantitativo, observacional y retrospectivo realizado a partir de datos recolectados de los resúmenes estadísticos mensuales (REM) del Programa de Salud Cardiovascular (PSCV) publicados por el Departamento de Estadística e Información de Salud (DEIS) del MINSAL. Para ello se verifican los REM desde el año 2008, excluyendo de este estudio los REM anteriores al 2011 por no cumplir con la calidad ni cantidad de datos suficientes para la construcción de la TC. Se realiza un recuento estadístico de pacientes bajo control del PSCV con diagnóstico de DM tipo I y II de 15 años y más, como de aque- llos que cuentan con FO vigente dentro del año calendario. Este análisis se realiza a nivel nacional, desagregado por SS y comunas. Se evidencian datos faltantes del REM del año 2017 pertenecientes al SS Coquimbo que se subsana con la solicitud de esta información vía transparencia.

La TC de FO, se entiende como el porcentaje de pacientes diabéticos del PSCV que cuenta con FO anual en un año calendario. La guía clínica de RD señala entre sus objetivos el screening de FO, la cual no distingue las diferencias conceptuales entre tamizaje y test diagnóstico de FO como lo hace por ejemplo el Reino Unido ${ }^{19}$. Además, tampoco se diferencian como prestaciones distintas en los REM. Para términos de estudio, el concepto de FO se utilizará en el mismo sentido de los REM, indistintamente si es examen diagnóstico o tamizaje. Entenderemos como FO aquel examen realizado de manera presencial o de forma remota a través de telemedicina por oftalmólogo o en su defecto, realizado por un TMO por medio de cámaras retinales.

Para el análisis se utiliza estadística descriptiva e inferencial. Se calculan las TC de los SS para cada uno de los años de estudio, así como la variación absoluta y relativa de las TC en el período 20112019. La tasa de variación absoluta, nos indica el incremento o decremento de la TC y se calcula por la diferencia entre la TC del 2011 y el 2019. La tasa de variación relativa significa la variación absoluta en términos porcentuales y es el cuociente entre la variación absoluta y la TC inicial (año 2011), multiplicado por 100 .

Además, se realiza una comparación de todos los sub-tramos continuos posibles de este período para la TC nacional (por ejemplo, del 2012 al 2019; del 2011 al 2014). También se comparan las TC anuales entre los SS para cada uno de los años del período. Para estos análisis de utiliza el test de hipótesis de Kruskal Wallis.

Para el análisis a nivel comunal, se calculan las TC para cada una de las 346 comunas y se agrupan en 100 intervalos de 1 punto porcentual (por ejemplo: intervalo de TC entre $0 \%$ y $<1 \%$; entre $49 \%$ $\mathrm{y}<50 \%$ ) para determinar la cantidad (frecuencia absoluta) de TC que contiene cada intervalo y su representación porcentual (densidad). Además, se analiza si existe asociación entre la población diabética comunal y la TC.

Para la administración de datos y análisis estadístico se realiza mediante el software R 3.6.2. 
Tasa de cobertura de fondo de ojo en Chile - R. Silva-Jorquera et al

\section{Resultados}

Análisis de la TC y variación a nivel nacional

La TC nacional comienza con 19,06\% en 2011 y alcanza su punto máximo el 2019 con $36,45 \%$, que representa una variación absoluta de 17,39 puntos porcentuales, y una variación relativa de $91,20 \%$
(Tabla 1). Entre las TC de los años 2011 al 2019 no se observan diferencias estadísticamente significativas (DES) (chi-cuadrado $=134,10, \mathrm{gl}=8$, p-value $<0,000)$. Sin embargo, desde el 2016 se evidencian DES en las TC (chi-cuadrado $=4,51$, $\mathrm{gl}=3, \mathrm{p}$-value $=0,211$ ), agudizándose desde el $2017($ chi-cuadrado $=1.49, \mathrm{gl}=2, \mathrm{p}$-value $=0,474)$

Tabla 1. Tasas de variación absoluta y relativa de las tasas de cobertura de fondo de ojo y comparación de la significancia estadística del período 2011-2019

\begin{tabular}{|c|c|c|c|}
\hline Servicio de Salud & $\begin{array}{l}\text { Variación Absoluta } \\
\text { (Diferencia entre TC } \\
\text { del } 2019 \text { y el 2011) }\end{array}$ & $\begin{array}{c}\text { Variación Relativa } \\
\text { (Diferencia porcentual al } \\
2019 \text { en relación con el 2011) }\end{array}$ & $\begin{array}{c}\text { Kruskal Wallis } \\
\text { p-value } \\
2011 \text { - } 2019\end{array}$ \\
\hline Viña del Mar Quillota & 38,85 & 489,37 & $0,000^{\mathrm{a}}$ \\
\hline Metropolitano Norte & 28,92 & 319,39 & $0,000^{\mathrm{a}}$ \\
\hline Metropolitano Sur & 27,37 & 182,8 & $0,019^{a}$ \\
\hline Metropolitano Oriente & 23,66 & 111,93 & 0,091 \\
\hline Metropolitano Occidente & 22,35 & 197,25 & $0,000^{\mathrm{a}}$ \\
\hline O'Higgins & 21,48 & 86,51 & $0,011^{a}$ \\
\hline Coquimbo & 19,99 & 84,16 & 0,372 \\
\hline del Reloncaví & 18,99 & 61,12 & $0,005^{\mathrm{a}}$ \\
\hline Antofagasta & 18,69 & 283,69 & $0,046^{a}$ \\
\hline Biobío & 17,77 & 59,29 & $0,000^{\mathrm{a}}$ \\
\hline Valparaíso San Antonio & 17,04 & 186,23 & 0,384 \\
\hline Araucanía Sur & 16,85 & 138,94 & 0,257 \\
\hline Arauco & 16,68 & 59,86 & 0,723 \\
\hline Osorno & 16,59 & 81,20 & 0,059 \\
\hline Maule & 16,26 & 95,19 & $0,005^{a}$ \\
\hline Aisén & 13,89 & 73,63 & 0,190 \\
\hline Valdivia & 13,30 & 45,16 & $0,000^{\mathrm{a}}$ \\
\hline Metropolitano Central & 12,46 & 198,51 & 0,201 \\
\hline Atacama & 11,51 & 144,85 & $0,035^{\mathrm{a}}$ \\
\hline Metropolitano Sur Oriente & 10,70 & 44,02 & $0,048^{\mathrm{a}}$ \\
\hline Talcahuano & 10,13 & 56,38 & 0,903 \\
\hline Ñuble & 8,29 & 20,65 & 0,498 \\
\hline Magallanes & 5,47 & 18,54 & 0,030 \\
\hline Chiloé & 5,24 & 21,37 & 0,758 \\
\hline Concepción & 4,09 & 15,59 & 0,270 \\
\hline Aconcagua & 3,87 & 16,13 & 0,970 \\
\hline Iquique & $-3,53$ & $-16,45$ & 0,223 \\
\hline Arica & $-5,16$ & $-16,71$ & 0,386 \\
\hline Araucanía Norte & $-8,42$ & $-24,21$ & $<0,001^{\mathrm{a}}$ \\
\hline Nacional & 17,39 & 91,21 & $<0,000^{a}$ \\
\hline
\end{tabular}

${ }^{a}$ Diferencias estadísticamente significativas $(p<0,05)$. Fuente: Elaboración propia en base a los resultados del estudio. 
(Figura 1). Cabe señalar que en el 2019 se registra $39,4 \%$ de hombres y $60,6 \%$ mujeres diabéticas. No es posible conciliar estos datos con precisión para aquellos que cuentan con FO.

\section{Análisis de la TC y variación por SS}

La mayor TC a nivel de SS se observa el año 2014 en el SS Metropolitano Sur Oriente (SSMSO) con $59,66 \%$, mientras que la menor se encuentra en el SS Metropolitano Central (SSMC) el año 2011 con $6,27 \%$. El SS Ñuble, alcanza la mayor TC anual promedio del período, mientras que el SSMC muestra la menor de ellas (Figura 2). Existen DES entre las TC de los 29 SS en todos los años del período analizados independientemente (p-value < 0,000). El SSMC encabeza los peores resultados en su TC en cuatro de los nueve años, lo sigue el SS Antofagasta en tres ocasiones, y los SS Iquique (SS Iquique) y SS Metropolitano Norte que alcanzan la TC mínima en una oportunidad cada uno. Al analizar cada SS independientemente a través del tiempo, se presentan DES en 13 de ellos (Tabla 1). Desde el 2017 ningún SS muestra DES en la TC, permaneciendo sin aumentos ni descen- sos significativos en este indicador. La diferencia entre la TC del 2019 y el 2011 alcanza su punto más alto es el SS Viña del Mar Quillota (SSVQ), mientras que la menor de ellas se presenta en el SS Araucanía Norte (SSANorte) (Tabla 1).

Contextualizando la situación en el año 2019, el SSMSO concentra la mayor población diabética, como también de aquellos que cuentan con FO. En contraste, el SS Aisén presenta la menor proporción de estos pacientes. La mayor TC se logra en el SS del Reloncaví (SSReloncaví), el cual, mantiene la TC sobre la barrera de $50 \%$ por seis años consecutivos desde el 2014. Por el contrario, el SS Iquique, junto con el SS Atacama y el SSMC, se posicionan con las peores TC, bajo la barrera del 20\% (Figura 3); valores similares a la TC nacional el año 2011 (Figura 2).

\section{Densidad y Frecuencia de las TC a nivel de municipios}

La densidad de las TC de los municipios va evolucionando a través del tiempo, comenzando con una distribución con una mayor proporción de municipios con bajas TC, hacia una distribu-

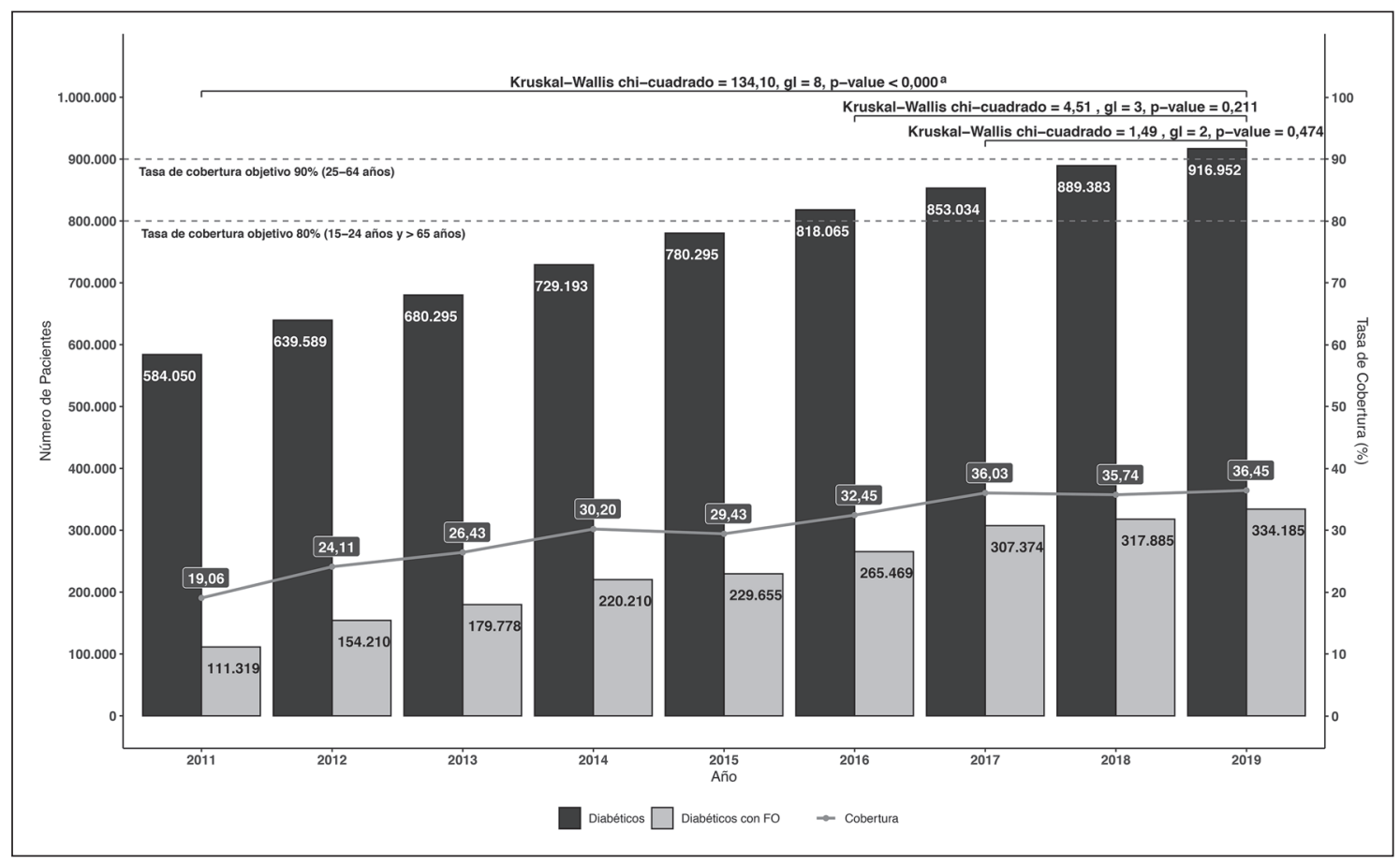

Figura 1. Tasa de Cobertura de fondos de ojo en Chile en el período 2011 al 2019. Nota al pie: Elaboración propia en base a información DEIS Serie P del 2011 al 2019. 
Tasa de cobertura de fondo de ojo en Chile - R. Silva-Jorquera et al

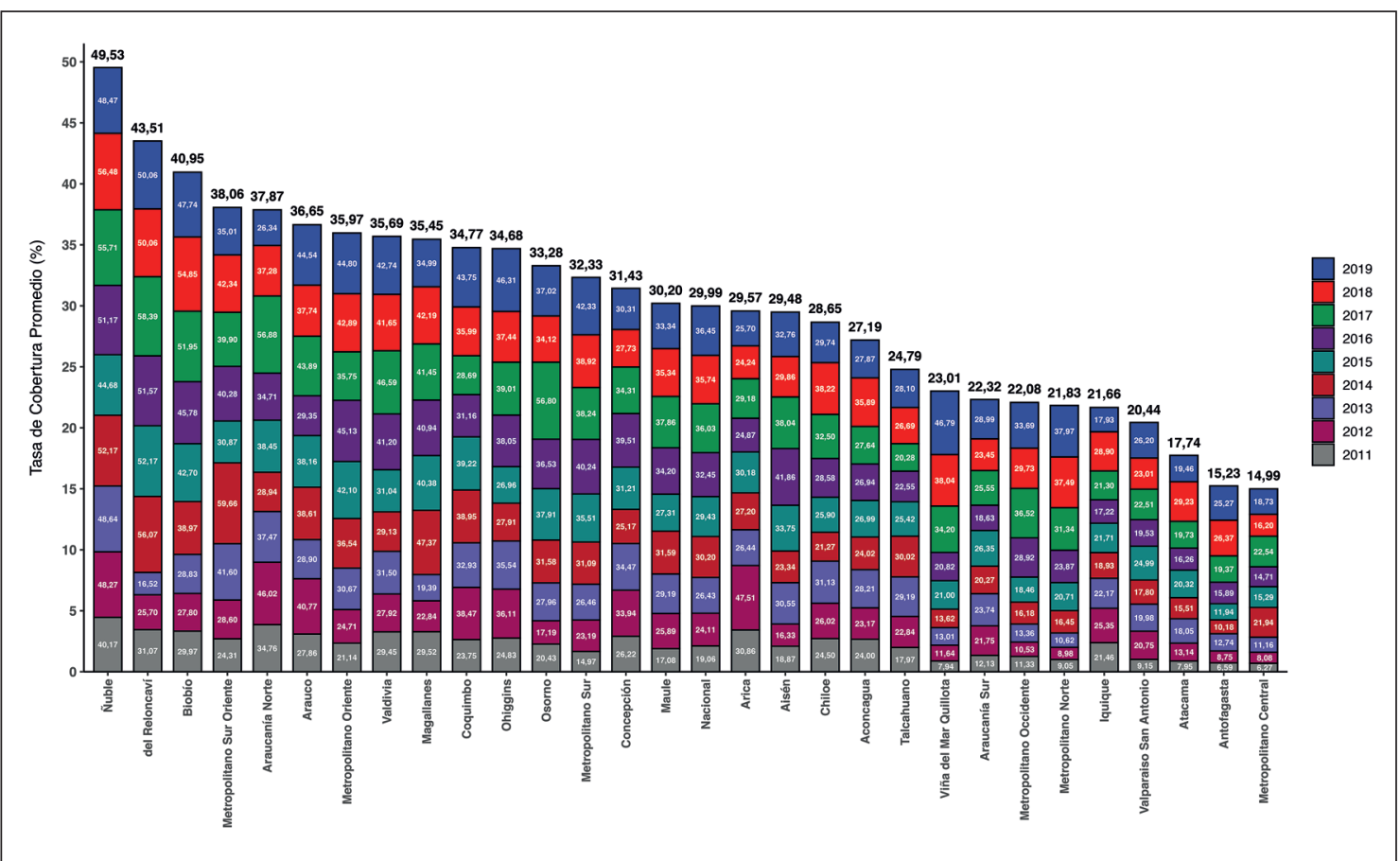

Figura 2. Tasas de cobertura anuales y anual promedio del período 20112019 desagregado por servicios de salud. Nota al pie: Elaboración propia en base a información DEIS Serie P del 2011 al 2019.

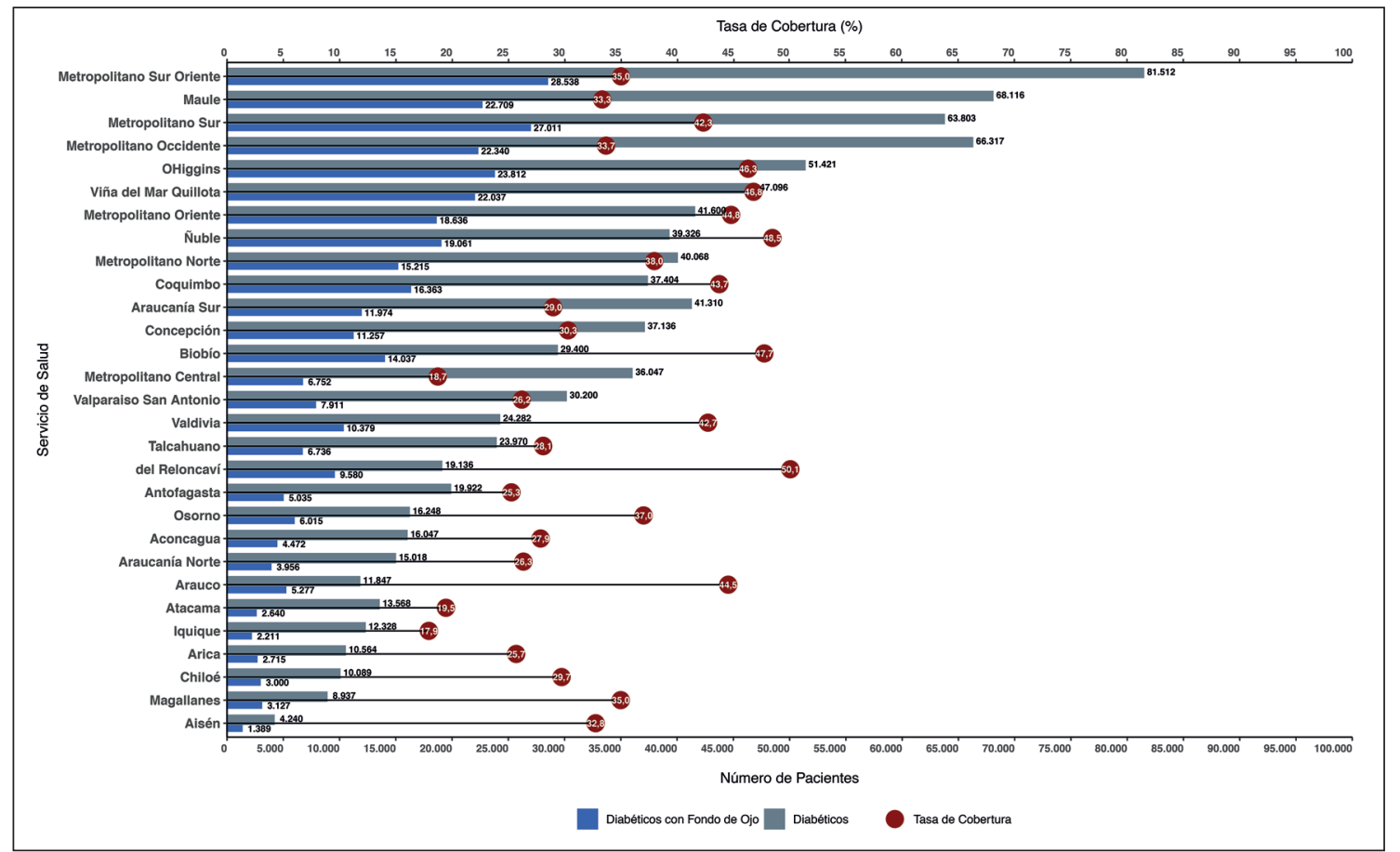

Figura 3. Población de pacientes con diabetes, de diabéticos con fondo de ojo anual vigente, y tasa de cobertura de fondo de ojo en el año 2019. Nota al pie: Elaboración propia en base a información DEIS Serie P del 2019. 


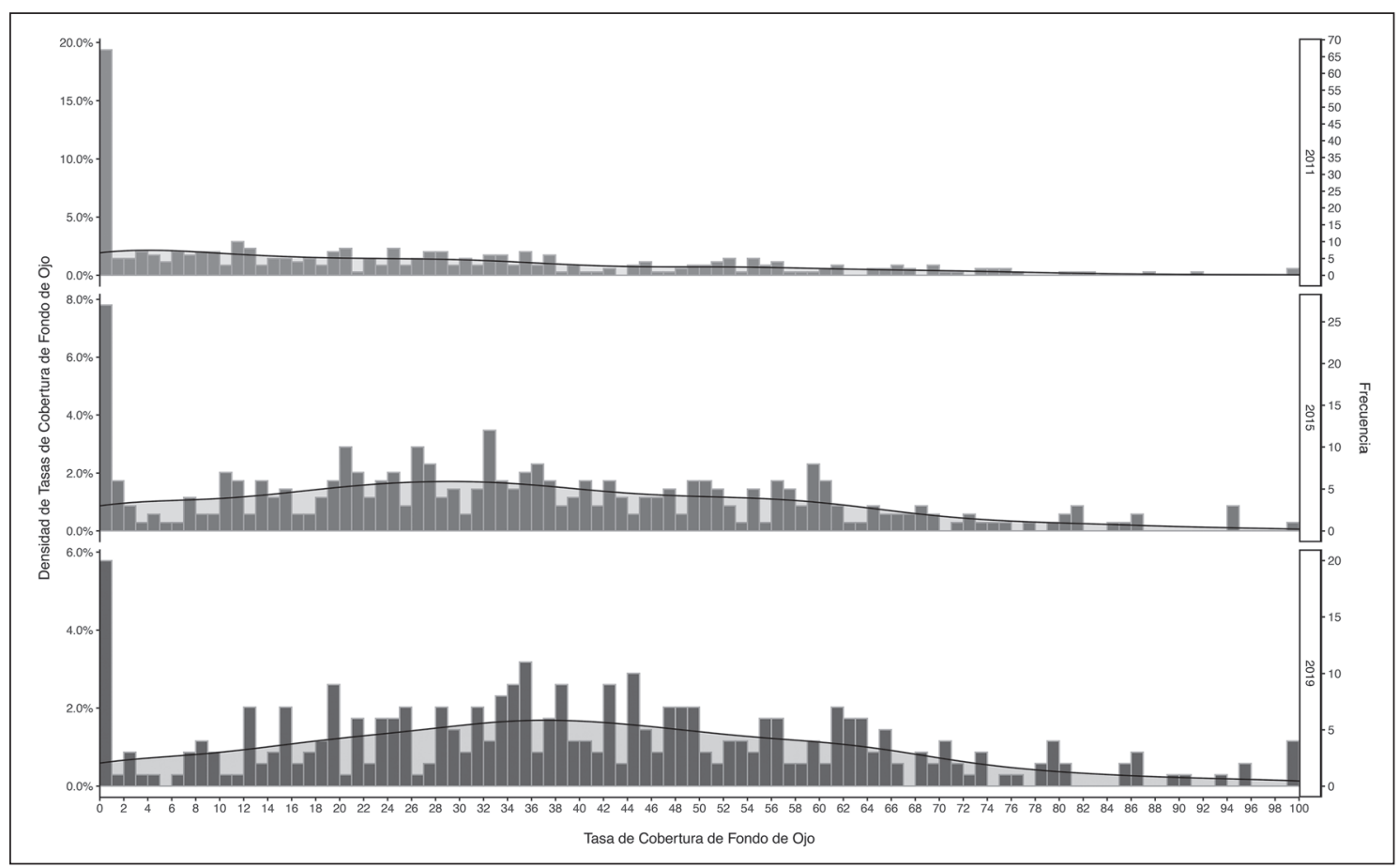

Figura 4. Frecuencia comunal de las tasas de cobertura de fondo de ojo en los años 2011, 2015 y 2019. Nota al pie: Elaboración propia en base a información DEIS Serie P del 2011, 2015 y 2019.

ción que desplaza la densidad de los intervalos de cobertura hacia mayores valores. La mayor cantidad de TC se ubican en el intervalo $0 \%$ y $<1 \%$ en cada uno de los años. En el 2011, existen 67 comunas en este rango (19,36\%), 27 de ellas en el 2015 (7,80\%), y 20 en el 2019 (5,78\%) (Figura 4). Por lo general son comunas con una baja población de personas con diabetes inscritas en el PSCV. En el 2019, 18 de las 20 comunas entre con TC entre $0 \mathrm{y}<1 \%$, tienen una población de diabéticos menor a 900 personas. Similar situación ocurre en comunas con la TC de 100\%; 4 comunas logran este valor el 2019, la mayor de ellas con una población de 48 pacientes diabéticos. A pesar de ello, no existe una asociación entre la población diabética comunal y la TC en el período de estudio $(\mathrm{r}(3112)=-0,083, \mathrm{p}<0,001)$.

\section{Discusión}

La meta de $80 \%$ y $90 \%$ de la TC propuesta por la autoridad sanitaria chilena ${ }^{16}$, está lejos de lograrse. A pesar de la introducción de inteligencia artificial para la predicción de RD en el 2018, no ha sido suficiente para aumentar la TC. Por otro lado, el ejemplo del SSVQ el cual no utiliza esta herramienta, ha aumentado su TC sostenidamente en el tiempo lo que podría relacionarse con la implementación de nueve UAPO a partir de 2013; dos de ellas móviles. Sería de especial interés estudiar el impacto de las UAPO en la TC, como también el real alcance para la realidad chilena, el uso de la actual herramienta de inteligencia artificial en el tamizaje de FO.

Por otro lado, este estudio evidencia una inequidad en el acceso a FO. Las mayores y menores TC, se mantienen en el tiempo por los mismos SS. Por ejemplo, el SS Iquique tiene una población diabética similar al SS Reloncaví, pero logra la mitad de la TC anual promedio de este último. Si bien es complejo realizar una comparación entre estos SS, ambos cubren a cinco comunas propuestas para ser declarados zonas rezagadas en materia social $^{20}$. Un estudio que caracteriza a centros de atención primaria en relación con su entorno, identifica inequidades geográficas de demanda insatisfecha como también una dificultad de co- 
nectividad entre el centro de salud y el transporte público; ${ }^{21}$ situación que podríamos plantearnos como una barrera de acceso al FO.

Si bien este estudio no evidencia una asociación entre la población diabética y la TC, las comunas con baja población diabética tienden a tener una TC muy baja, o bien demasiado alta. Por otro lado, en el año 2019 la comuna de Cerrillos y la de Estación Central pertenecientes al SSMC, no logran superar $2 \%$ en la TC. Aquí evidenciamos que comunas pertenecientes al SS "capital" de Chile presentan un deficiente acceso a FO. En cualquier circunstancia, es primordial subsanar las TC de las comunas más rezagadas.

Una de las mayores limitantes para el aumento de la TC es la inconsistencia administrativa de la garantía GES en la relación DM RD, considerando que todo beneficiario diabético con sospecha de $\mathrm{RD}$, tendrá la garantía de acceder a una confirmación diagnóstica dentro de 90 días. ${ }^{22}$ En la práctica, se genera un tamizaje de FO focalizado, toda vez que solamente acceden al tamizaje de FO aquellos pacientes diabéticos que administrativamente (apertura de caso sospecha de problema de salud GES, con su respectiva solicitud de interconsulta) cuentan con una sospecha diagnóstica de RD. Los demás pacientes diabéticos quedan segregados del tamizaje, provocando una inequidad en el acceso y prevención de la RD. Exitosos resultados bajo la modalidad de invitación telefónica o por correo certificado al tamizaje ha tenido Inglaterra ${ }^{19} \mathrm{y}$ Finlandia ${ }^{23}$.

Aunque Chile ha transitado desde el FO presencial hacia la modalidad de telemedicina, aún se posiciona lejos de los niveles de cobertura alcanzados en algunos países recientemente. Inglaterra obtiene $82,4 \%{ }^{19}$ en su último reporte anual; Finlandia $78 \%{ }^{23}$; Gales alcanza $67,5 \%{ }^{24}$; mientras que Canarias, logra una TC de $56,82 \%{ }^{25}$. Por otro lado, no se ha logrado obtener evidencia de las realidades de las TC en Latinoamérica para establecer una comparación con los países vecinos. Si bien pudiera no ser adecuado realizar comparaciones entre la realidad chilena con la del Reino Unido, Inglaterra ha logrado erradicar la RD como primera causa de ceguera en edad laboral ${ }^{19}$, convirtiéndose en un modelo a seguir en esta materia.

Es así como Chile, a pesar de lograr la mayor TC de FO histórica el año 2019, presenta una baja TC para los objetivos propuestos e inequidad entre las TC de los SS y comunas. Adicionalmente, se evidencia un estancamiento en la evolución de la TC desde el año 2016, por lo que necesitan estrategias que subsanen esta realidad, acompañado de un sólido componente educativo. Para ello se entregan las siguientes recomendaciones:

- Crear un plan de tamizaje de FO formal y sostenible en el tiempo.

- Es deseable una actualización de la Guía Clínica de RD que data del 2010, que clarifique en su totalidad esta materia.

- Chile necesita mejorar sus registros estadísticos ${ }^{26}$, que considere entre otros aspectos, una distinción entre el registro de tamizaje de FO preventivo para pacientes diabétcos y el examen de FO para aquellos que han sido previamente diagnosticados con $\mathrm{RD}$.

- Es primordial para fortalecer la investigación y vigilancia epidemiológica, establecer una homologación de los grupos etáreos en los registros de pacientes diabéticos, con aquellos que cuentan con FO. Existen 14 grupos etáreos para el registro de diabéticos y dos para los que cuentan con FO.

- Eliminar todas las barreras administrativas que obstaculicen el acceso al tamizaje de FO, invitando abiertamente a los pacientes diabéticos a someterse al tamizaje anual.

- Reforzar la fuerza de trabajo mediante la implementación de UAPO fijas y móviles, equipadas con cámaras retinales.

- Se sugiere una revisión acerca de los reales alcances para la realidad chilena de la inteligencia artificial utilizada en la actualidad.

- Por último, estos esfuerzos deben acompañarse con la entrega de tratamiento oportuno a pacientes diagnosticados con $\mathrm{RD}$, por lo que la implementación de centros láser que absorban el aumento en la TC debe ser un pilar fundamental para evitar la ceguera como consecuencia de la diabetes.

Por último, esta medición cruda de cobertura es un aporte para mejorar la medición de la cobertura universal en salud propuesto por Naciones Unidas en su objetivo de desarrollo sostenible $3,8^{27}$. Como desafío de investigación posterior, es necesario conocer la cobertura efectiva del tamizaje de FO en los pacientes diabéticos, que representaría la proporción de la potencial ganancia en salud en relación con lo experimentado ${ }^{28}$. 


\section{Referencias}

1. World Health Organization. World Health Statistics 2014 [Internet]. 2014 Available from: https:// apps.who.int/iris/bitstream/handle/10665/112738/ 9789240692671_eng.pdf;jsessionid=4DF0CB66224C6D00C80CD77EC71A8CEC? sequence $=1$ [citado el 23 de julio de 2020].

2. GBD 2019 Diseases and Injuries Collaborators. Global burden of 369 diseases and injuries in 204 countries and territories, 1990-2019: a systematic analysis for the Global Burden of Disease Study 2019. Lancet 2020; 396(10258): 1204-22.

3. Federación Internacional de la Diabetes. Atlas de la diabetes de la FID [Internet]. Vol. 9. 2019 Available from: https://diabetesatlas.org/upload/resources/material/20200302_133352_2406-IDF-ATLAS-SPAN-BOOK. pdf [citado el 28 de diciembre de 2020].

4. Wong TY, Sun J, Kawasaki R, Ruamviboonsuk P, Gupta $\mathrm{N}$, Lansingh VC, et al. Guidelines on Diabetic Eye Care. Ophthalmology. 2018; 125 (10): 1608-22.

5. Photocoagulation for Diabetic Macular Edema: Early Treatment Diabetic Retinopathy Study Report Number 1 Early Treatment Diabetic Retinopathy Study Research Group. Arch Ophthalmol. 1985; 103(12): 1796-806.

6. Relhan N, Flynn HW. The Early Treatment Diabetic Retinopathy Study historical review and relevance to today's management of diabetic macular edema: Curr Opin Ophthalmol. 2017; 28(3): 205-12.

7. Rami HE, Barham R, Sun JK, Silva PS. Evidence-Based Treatment of Diabetic Retinopathy. Semin Ophthalmol. 2017; 32 (1): 67-74.

8. Shah CP, Chen C. Review of Therapeutic Advances in Diabetic Retinopathy. Ther Adv Endocrinol Metab. 2011; 2(1): 39-53.

9. Abuauad S, Guzmán P, Urzúa C. Prevalencia de retinopatía diabética y edema macular en población diabética del CESFAM Cordillera Andina de Los Andes. Revista Chilena de Salud Pública 2014; 18(1): 81-6.

10. Avendaño-Veloso A, Parada-Hernández F, González-Ramos R, Dougnac-Osses C, Carrasco-Sáez JL, Scanlon PH. Teleophthalmology: a strategy for timely diagnosis of sight-threatening diabetic retinopathy in primary care, Concepción, Chile. Int J Ophthalmol. 2019; 12(9): 1474-8.

11. Covarrubias T, Delgado I, Rojas D, Coria M. Coverage of a screening program and prevalence of diabetic retinopathy in primary care. Rev Med Chile 2017; 145(5): 564-71.

12. Ministerio de Salud [Internet]. Santiago: Problemas de
Salud - AUGE 85 - 2019. Available from: https://auge. minsal.cl/problemasdesalud/index [citado el 23 de febrero de 2020].

13. Fondo Nacional de Salud. [Internet]. Santiago: Conoce Fonasa 2019. C2019. Available from: https://www.fonasa.cl/sites/fonasa/conoce-fonasa [citado el 22 de julio de 2020].

14. Ministerio de Salud [Internet]. Santiago: Guía Clínica Retinopatía Diabética. c2010. Available from: https:// www.minsal.cl/portal/url/item/7222754637ab8646e04001011f014e64.pdf [citado el 22 de junio de 2020].

15. Flores R, Donoso R, Anguita R, Flores R, Donoso R, Anguita R. Management of diabetic retinopathy using telemedicine and network integration. Rev Med Chile 2019; 147 (4): 444-50.

16. Subsecretaría de Redes Asistenciales. Matriz de Cuidado a lo Largo del Curso de la Vida [Internet]. 2020. Available from: https://www.minsal.cl/wp-content/ uploads/2020/09/20200908_MATRIZ-DE-CUIDADOSA-LO-LARGO-DEL-CURSO-DE-VIDA.pdf [citado el 28 de diciembre de 2020].

17. Public Health England. NHS Screening Programmes in England 2017 to 2018. 2019 Mar p. 17.

18. Ministerio de Salud [Internet]. Santiago: Guía de Práctica Clínica - Problema de Salud AUGE N³1, Retinopatía Diabética. Available from: https://diprece.minsal.cl/ le-informamos/auge/acceso-guias-clinicas/guias-clinicas-desarrolladas-utilizando-manual-metodologico/ retinopatia-diabetica/descripcion-y-epidemiologia/ [citado el 22 de julio de 2020].

19. Scanlon PH. The English National Screening Programme for diabetic retinopathy 2003-2016. Acta Diabetol. 2017;54 (6): 515-25.

20. Ministerio del Interior [Internet]. Santiago: Subsecretaría de Desarrollo Regional - Informe Comunas Susceptibles Zonas Rezagadas 2020. Apr 2020. Available from: http://territoriosdeconvergencia.subdere.gov. cl/files/doc_zonas_rezagadas/ORD\%201238\%20-\%20 Informe\%20Comunas\%20Susceptibles\%20Zonas\%20 Rezagadas\%202020.pdf [citado el 29 de junio de 2020].

21. Pietri DD, Dietrich P, Mayo P, Carcagno A. Indicadores de accesibilidad geográfica a los centros de atención primaria para la gestión de inequidades. Rev Panam Salud Publica. 2013;9.

22. Ministerio de Salud. Problemas de Salud 31 Retinopatía Diabética - AUGE 85 [Internet]. 2020. Available from: https://auge.minsal.cl/problemasdesalud/index/31 [citado el 3 de enero de 2020].

23. Hautala N, Aikkila R, Korpelainen J, Keskitalo A, Kurikka A, Falck A, et al. Marked reductions in visual impairment due to diabetic retinopathy achieved by 
efficient screening and timely treatment. Acta Ophthalmologica 2014; 92 (6): 582-7.

24. Screening Division of Public Health Wales [Internet]. DESW Annual Statistical Report 2018-19. Feb 2020. Available from: https://phw.nhs.wales/services-and-teams/screening/diabetic-eye-screening-wales-desw/diabetic-eye-screening-wales-annual-statistical-report-2018-19/diabetic-eye-screening-wales-annual-statistical-report-2018-19/[citado el 29 de junio de 2020].

25. Dirección general de programas asistenciales, Servicio Canario de la Salud. Memoria Proyecto Retisalud: Cribado de la retinopatía diabética mediante la retinografía no midriática en atención primaria [Internet]. 2017. Available from: https://www.mscbs.gob.es/organizacion/ sns/planCalidadSNS/pdf/BBPP_2017/BBPP_Diabe-
tes_2017/BBPP_DIABETES_Canarias.2017.pdf [citado el 2 de enero de 2020].

26. Covarrubias T, Oyarte M, Cabieses B, Coria M. Chile necesita mejores estadísticas y mayor cobertura en tamizaje de retinopatía diabética. Rev Med Chile 2017; 145(12): 1633-5.

27. Ng M, Fullman N, Dieleman JL, Flaxman AD, Murray CJL, Lim SS. Effective Coverage: A Metric for Monitoring Universal Health Coverage. PLOS Medicine. 2014; 11(9): e1001730.

28. United Nations. Transforming our world: the 2030 Agenda for Sustainable Development, New York [Internet]. United Nations; 2015. Available from: https://documents-dds-ny.un.org/doc/UNDOC/LTD/ N15/285/76/PDF/N1528576.pdf?OpenElement[citado el 3 de enero de 2020].

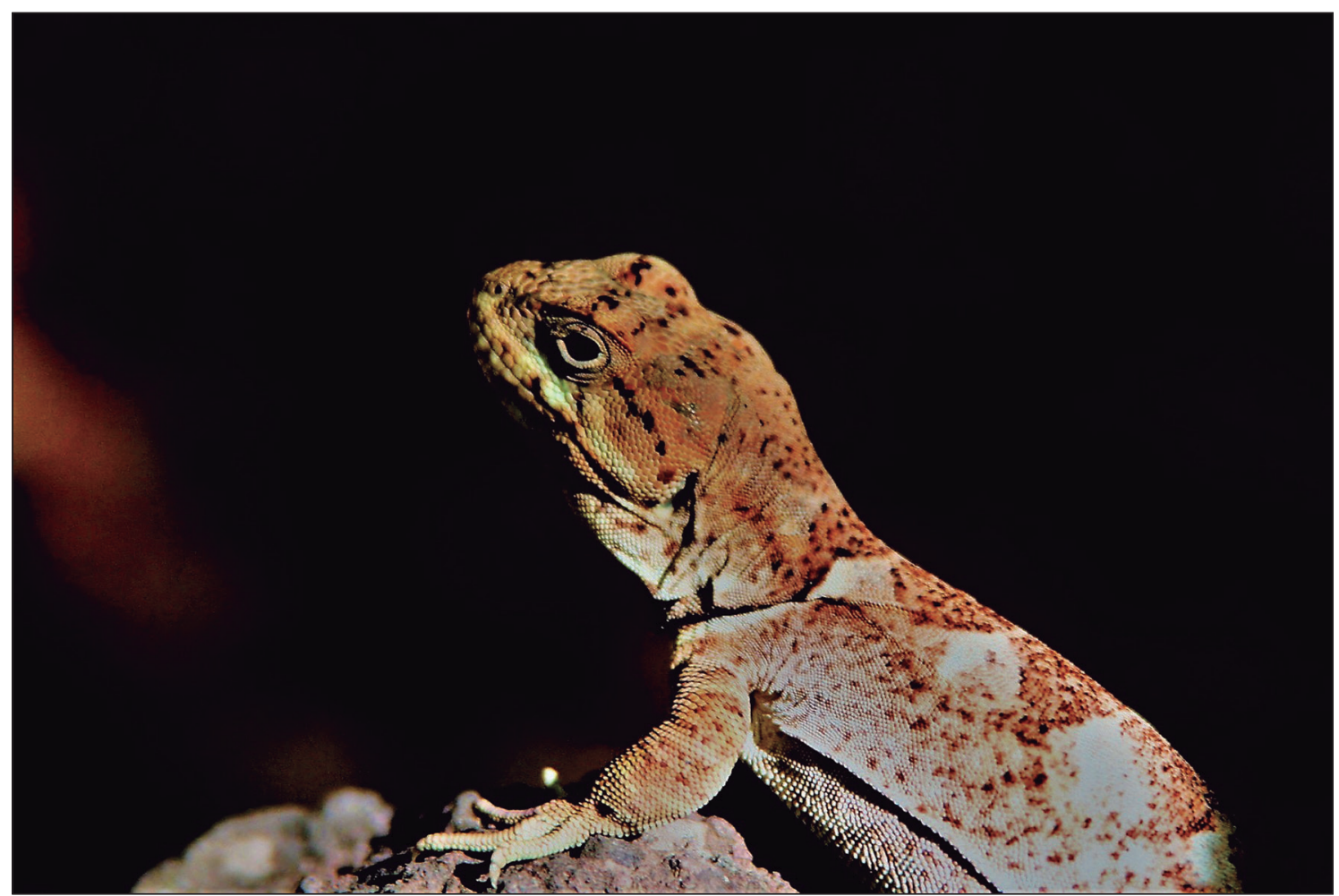

Lagarto Gruñido, Radal 7 Tazas, Región Maule. Dr. Jorge Sapunar Zenteno 